\title{
Automated Reconfiguration Enabled Mesh Network based on Fuzzy Logic for Performance Improvement
}

\author{
Vijaykumar Naik Pawar \\ M.Tech., Dept of CSE \\ KLS Gogte Institute of Technology \\ Udyambag, Belagavi, Karnataka, India
}

\author{
Prof. Vidya R. Kulkarni \\ Associate Professor, Dept of CSE \\ KLS Gogte Institute of Technology \\ Udyambag, Belagavi, Karnataka, India
}

\begin{abstract}
Wireless Mesh Networks (WMN) is a new exciting technology that is expected to overcome the limitations and to improve the performance of various wireless networks such as adhoc networks, WLANS, WPANS, and WMANs. Heterogeneous Mesh networks running on different technologies with different channels, which is commonly known as multi radio network will be dominant in the nextgeneration wireless networks with the integration of various wireless access networks. In such a network, obtaining routes that elongates the QoS and extends the route lifetime without compromising on performance issues plays a significant role.

There is a need to enhance the performance in WMN during dynamic link failures. This paper presents a self reconfiguration wireless mesh network that dynamically searches for the best available path among the available multiple paths to the internet. The best path will be selected based on the fuzzy rule set. The ARS with fuzzy logic results in improved throughput and latency.
\end{abstract}

\section{Keywords}

Wireless mesh networks, Self Reconfiguration, Multiradio wireless, IEEE 802.11, Fuzzy logic.

\section{INTRODUCTION}

Wireless Mesh Networks (WMNs) is a heterogeneous network of mesh routers and mesh clients, where mesh routers form the backbone of network and with minimum movement whereas client can either stationary or might have mobility . They provide network access for both mesh and conventional clients. Mesh routers have bridging and gateway functionality that in turn helps in the integration of WMNs with other existing networks such as internet, cellular, IEEE 802.15, IEEE 802.16, sensor networks, etc. In WMN each node operates not only as a host, but also as a router, forwarding packets on behalf of other nodes that may not be within the scope of direct wireless transmission of the destinations.[1]

WMN supports larger applications and also provides several benefits to users such as no wiring cost, automatically getting connected to all nodes, network flexibility and ease of installation and also discovers new routes automatically. This makes WMN preferable in fast and easy deployment of technology.

A WMN is dynamic self-organized and self-configured, where the nodes of the network automatically set and maintain mesh connectivity among them (creating, in effect, an ad hoc network). This feature provides many advantages to $\mathrm{WMN}$ as low initial cost, easy network maintenance, robustness, and reliable service coverage.
Obtaining an optimal path in a mesh network and then maintaining QoS is always a challenge mainly because of two factors. First, the demand for bandwidth is uneven i.e. a node watching a YouTube video will require more bandwidth then a node just surfing a HTML file. On the other hand, if a node is transmitting a song or video to another device without being connected to the Internet, then the demand will be different. More bandwidth demand leads to collision and thus reduces the signal to noise ratio. So, instead of transmitting long way in one session, nodes are better off to flexible connectivity where they can choose the peer who wants to connect to on the fly.

Link failures in WMN occur frequently and results in server performance degradation. Several techniques have been proposed for link failure recover in wireless mesh network. First technique is the, resource-allocation algorithms, in this method the initial planning for resources of network are done. These can provide the best configuration plan but this requires global configuration changes which are unattractive in case of frequent link failure [2]. Second is the, a greedy channelassignment algorithm can reduce the need for changes to the network reconfiguration by changing only the faulty links that is in greedy channel assignment the reconfiguration is not done on the entire network instead only faulty links are changed[4]. Next is the, fault-tolerant routing protocol, such as local rerouting or multipath routing can be adopted for avoiding the faulty links. However, these methods depend on detour paths or redundant transmissions, which may require more network resources than link-level network reconfiguration. Whenever a link failure occurs the routing protocol can route the packet via a different link.

A research by kyu-Han kim and Kang G.shin suggested a Self Reconfiguration system where the node without dropping the call decides to which node it wants to get connected to. Self Reconfiguration is studied extensively for Mesh network. In a Self Reconfiguration a node decides the peer it wants to get connected to. Even if the radio link quality observed at a node with an access point is good, the access point may itself suffer from packet congestion due to too many connections. [3]

Our contribution in the work is twofold: Firstly we propose a unique admission control based routing which is a variant of DSDV protocol where a router participates in routing only if it has sufficient bandwidth to meet the demand. Secondly we offer a unique fuzzy based solution by incorporating bandwidth and delay metrics to resolve the best available connection for a node and update the route on the fly by changing the connected access point/ base station through appropriate handoff which results in auto reconfiguration. This mechanism is called automated reconfiguration. 
The rest of the paper is organised as follows. Section II describes the related work, section III describes ARS architecture, section IV describes system implementation and experimentation an $\mathrm{d}$ section $\mathrm{V}$ concludes the paper.

\section{RELATED WORK}

\subsection{Network Model and Assumptions}

Multiradio WMN: A multiradio wireless network is where there are more than one access point. There are multiple nodes, multiple access point can communicate with each other and then get connected to the gateway. Here mostly channel related link failures are due to the lack of bandwidth, lack of energy drainage of nodes or due to lack of very high power.[6]

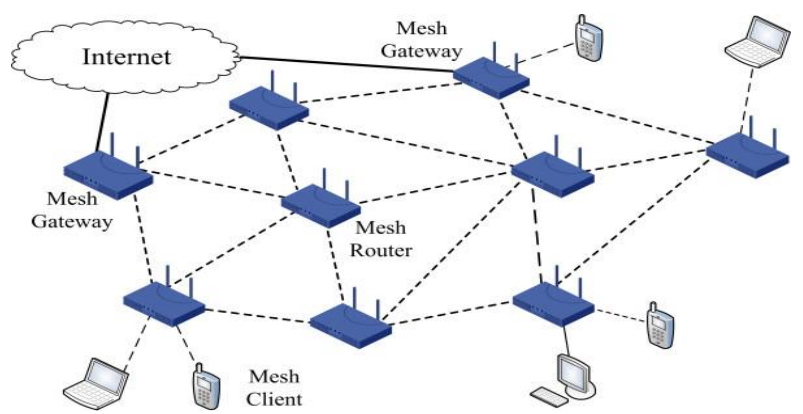

Fig 1: Multiradio WMN

QoS Support: In operation, each mesh node periodically sends its local channel usage and quality information for all outgoing connections via management messages to the gateway control. The gateway uses this information for admission control.

QoS-Awareness: Automated Reconfiguration must meet required QoS constraints on each link as much as possible. First, given the limited bandwidth of each link's existing channel assignment and scheduling algorithms can provide about optimal network configurations. However, as noted above, these algorithms may require global network configuration changes with the changing demands of local service quality, thus causing disruptions. We need a reconfiguration algorithm that incurs only local changes and increase the most of the opportunity to respond to the demands of service quality.

Cross-Layer Interaction: Network reconfiguration must jointly consider the network settings across multiple layers. In the network layer, routing protocols with fault tolerance, such as changing local path or multipath routing allow reconfiguration flow to meet the quality of service restrictions by exploiting path diversity. However, consume more network resources than link reconfiguration due to its dependence on roundabout route or redundant transmissions. In the other manual tasks, channel and link through the network and link layers can avoid the overhead of diversion, but they must be taken to avoid interference faults as additional service of neighbouring nodes.[4]

\subsection{Need for Self Reconfiguration}

- Recovering from link-quality degradation: Several wireless networks such as Wifi, Bletooth might be operating on the same channels or adjacent channels, due to this severe interference it leads to link quality degradation between the nodes in WMN's. This problem can be solved using the concept of channel switch. By changing the tuned channel link to other channels which is interference-free, local link recovery can be achieved.
- Satisfying dynamic QoS demands: Links in some areas, such as conference rooms may not be able to accommodate increased demands for quality of service to end users. I.e. links around a conference room can have too much traffic to relay data / video during the session. In order to provide large amounts of data in real time the concept of radio switched can be used. This is accomplished by reallocating highly loaded radio to less loaded radius radios.

- Coping with heterogeneous channel availability: Links in some areas may not be able to access the wireless channel for a certain period of time, because of spectrum regulation. For example, some links in a WMN need vacate current channels if channels are being used for emergency response near wireless links. These links may seek alternative channels available in the same area.

\section{ARS ARCHITECTURE}

Automated reconfiguration enabled wireless mesh network is a distributed system that is easily deployable in IEEE 802.11based mr-WMNs. Running in each mesh node; ARS supports self-reconfigurability via the following features. [5]

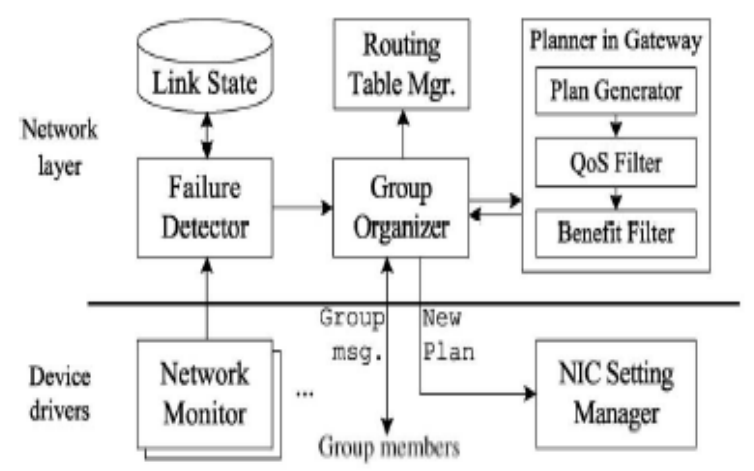

Fig 2: ARS Architecture

-Localized reconfiguration: Since multiple radio's and channels are available, during a link failure system generates a reconfiguration plans locally. The reconfiguration plan changes the network configurations only near the faulty area whereas keeping the network configuration at the remote locations unchanged.

- Cross-layer interaction: For generating the reconfiguration plan's the ARS actively interact through the network and link layers. This interaction allows ARS to include a change of route in reconfiguration along with link layer reconfiguration. ARS can also maintain connectivity during the recovery period using the routing protocols.[4]

- QoS-aware planning: ARS initially will keep on monitoring the state of the network. Whenever there is a link failure the system immediately looks for a reconfiguration plan which satisfies the required QoS. And pointed out earlier most of the algorithms require network configuration to be changed globally; this system only incurs local reconfiguration plans.

\subsection{Algorithm}

1. calculate Etx at each node with the of probing hello packets.

\section{Generate RREQ packet. Append needed bandwidth}

3. Nodes receiving RREQ forwards the request only if it's available $\mathrm{BW}>$ demand bandwidth. 
4. Before forwarding node appends it's bandwidth in RREQ packet.

5. At destination average BW is calculated. Packet delay is calculated from Receive time-Sending Time.

The best route is selected based on RouteQuality. Quality=FuzzyOut(BW,Delay).

6. Destination node replies by transmittig RREP packet. While transmission some nodes may overhear this packet that is not intended for it. This accounts to interference and the overheared packet is dropped.

7. Multiple paths are obatined and the path with high cost (Quality)is selected .

8. Data is transmitted over the selected path

9. When the nodes move, mobility model sends message to physic layer.

10. Physic layer initiates channel quality assessment by looping through the signal from all the access point.

11. Physic layer finds the access point with base station and request for a handoff which results in auto reconfiguration.

12. Once the access point is switched, path is changed locally through new access point.

13. For routing, flood control technique is adopted by restricting the route hops bellow 5 .

14. This mechanism is compared with present DSDV based system where only admission control is employed for QoS.

ARS at each mesh node monitors the quality of outgoing wireless links and reports the results to a gateway via a management message. Second, once a fault in a link is detected, ARS at the detector node (s) triggers the formation of a group between local network routers that use a faulty channel, and one of the members group has been chosen as a leader with well -known bully algorithm. Third, the leader node sends a message to request planning at the gateway. Then, the gateway requests are synchronized if there are multiple planning requests and generates a reconfiguration plan .Fourth, the gateway sends a reconfiguration plan to the leader node and group members. Finally, all nodes in the cluster run the appropriate configuration changes, if any, and resolve the group.

\section{SYSTEM IMPLEMENTATION AND EXPERIMENTATION}

We have implemented ARS using Omnetpp3.3p2 network stimulator. OMNeT++ is an object oriented, modular discrete network simulator. Developed in 1998, it is still new product. It is an open source package. This simulation package built based on C++ language foundation, it offers class library, GUI support (animation of network environment and graphical network editing). This kind of simulator can be used for modelling queuing networks, validating hardware architectures, traffic modelling and to measure evaluation performance of complex systems. OMNeT++ package specifically designed for telecommunication networks, it also gives good simulation performance and analytical results for sensor networks, mesh networks and several wireless network types. OMNeT++ is still young software package. It provides pre-built modules but less in number compared other simulators. OMNeT++ is carefully designed in such a way that it is easy, flexible, well organized and user-friendly than other simulators. OMNeT++'s internal architecture is model based which comprises of several hierarchical overlapping modules. The depth of overlapping of modules is limited to some degree and permits users to reflect logical components of actual system in module form. Modules in the simulator communicate among themselves via message passing, a module can send message directly to the destination or follow pre-defined path through gates and connections which possess properties like delay, bandwidth, and error rate. At the time of simulation run, simple modules are capable to run in parallel mode, since they are implemented in co-ordination with $\mathrm{C}++$ programming code. To create a simple module user can create objects with help of OMNeT GUI and also need to write subsequent $\mathrm{C}++$ programming code to represent objects that are created in OMNeT GUI.

\subsection{Implementation Details}

Fig. 2 shows the software architecture of ARS. Initially the ARS will keep on monitoring the state of the network using the network monitor. All the routes satisfying the required QoS will be stored in the routing table; these entries are used during the network reconfiguration. The failure detection module detects any of the link failure in the network and maintains the link state table. And the group organizer forms a local group among the mesh networks.

The module in this driver includes:1) Network monitor, monitors link-quality and is extensible to support as many multiple radios as possible and 2) NIC manager, which effectively reconfigures NIC's settings based on a reconfiguration plan from the group organizer.

\subsection{Fuzzy Based System}

When the system generates the route reply packets, the paths on which these packets would be send will depend on the fuzzy rule set. The block diagram of the fuzzy system is as shown in the figure 3 . The first step is the fuzzification where the numeric values (ie of bandwidth and delay) are converted into fuzzy values and then each nodes checks for bandwidth and delay values with the reference values. Then bandwidth and delay will be classified as low, medium or high. The fuzzy rule set table is created based on the fuzzy values and it generates a output which is used in the decision system.[5] 


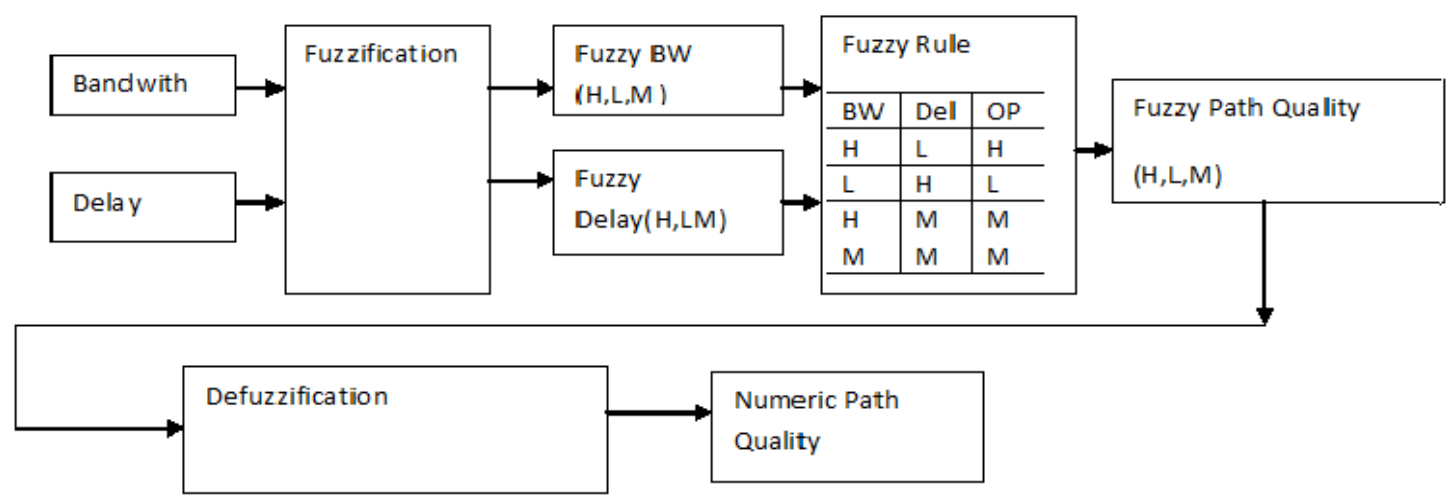

Fig 3 : Fuzzy System

\subsection{Experimental Setup}

To evaluate our implementation, we have first constructed a heterogeneous multi hop wireless mesh network. There are a total of 35 nodes over an area of $950 * 950$. One of the node is assumed to be a internet node. Objective of all the other nodes is to get connected with the internet. The source node will be transmitting data from the application layer, which will go routing layer(all routing table activities such as RREQ, RREP, RERR, hello packets) are done over here. And that would go to MAC layer and then move to physical layer. The Mobility to any node will be done by the mobility layer, which changes the $\mathrm{X}$ and $\mathrm{Y}$ location of the nodes.

\subsection{Experimental Results}

We evaluated the improvements achieved by ARS using fuzzy logic, including throughput and packet delivery ratio, latency and control overhead.

1) Throughput: Throughput is the ratio of message length and latency. We also set the bandwidth requirement of every link to $20 \mathrm{Mb} / \mathrm{s}$ and delay of $12 \mathrm{~ms}$. The graph shown in the figure 4 , number of nodes in the WMN is compared with throughput. Generally throughput is measured in terms of Mbps. Throughput of proposed work is higher than present system. The graph shown in the figure 5, Packet Rate in the WMN is compared with throughput. Generally throughput is measured in terms of Mbps. Even when the packet rate is varied the throughput is higher in ARS with fuzzy logic. TP_pres represents throughput gains without fuzzy logic whereas TP_pros represents Throughput gain with fuzzy logic. The maximum throughput for proposed system as number of active nodes increase throughput increases gradually but in case existing system throughput decreases as number of nodes increases.

2) Latency: Latency is defined as the end to end delay between transmitter and receiver. Latency is typically measured in milliseconds. the average latency of ARS without fuzzy logic was $0.1281 \mathrm{~ms}$ whereas with fuzzy bandwidth and delay it is $0.0470 \mathrm{~ms}$. As shown in the graph figure 6 , number of nodes versus latency (delay) is compared. Present system involves large latency but in case of proposed system latency is less, due to minimized latency accuracy of the transmission increases. Also as shown in the graph figure 7, Packet Rate versus latency (delay) are compared. Present system involves large latency but in case of proposed system latency is less, due to minimized latency accuracy of the transmission increases. Latency_pres represents Latency without fuzzy logic whereas Latency_pros represents Latency with fuzzy logic. Present system involves large latency but in case of proposed system latency is less, due to minimized latency accuracy of the transmission increases.

Table 1: Throughput values with varying number of nodes

\begin{tabular}{|c|c|c|}
\hline Node & $\begin{array}{c}\text { ARS } \\
\text { Throughput in } \\
\text { Bps }\end{array}$ & $\begin{array}{c}\text { ARS with fuzzy logic } \\
\text { Throughput in Bps }\end{array}$ \\
\hline 70 & 596947 & $1.55 \mathrm{E}+06$ \\
\hline 65 & 767160 & $1.42 \mathrm{E}+06$ \\
\hline 60 & 920157 & $1.42 \mathrm{E}+06$ \\
\hline 55 & 969436 & $1.45 \mathrm{E}+06$ \\
\hline 50 & $1.32 \mathrm{E}+06$ & $2.01 \mathrm{E}+06$ \\
\hline 45 & $1.46 \mathrm{E}+06$ & $1.43 \mathrm{E}+06$ \\
\hline 40 & $1.57 \mathrm{E}+06$ & $1.43 \mathrm{E}+06$ \\
\hline 35 & $6.02 \mathrm{E}+05$ & $1.46 \mathrm{E}+06$ \\
\hline 30 & $1.11 \mathrm{E}+06$ & $1.44 \mathrm{E}+06$ \\
\hline 25 & $9.51 \mathrm{E}+05$ & $1.45 \mathrm{E}+06$ \\
\hline
\end{tabular}

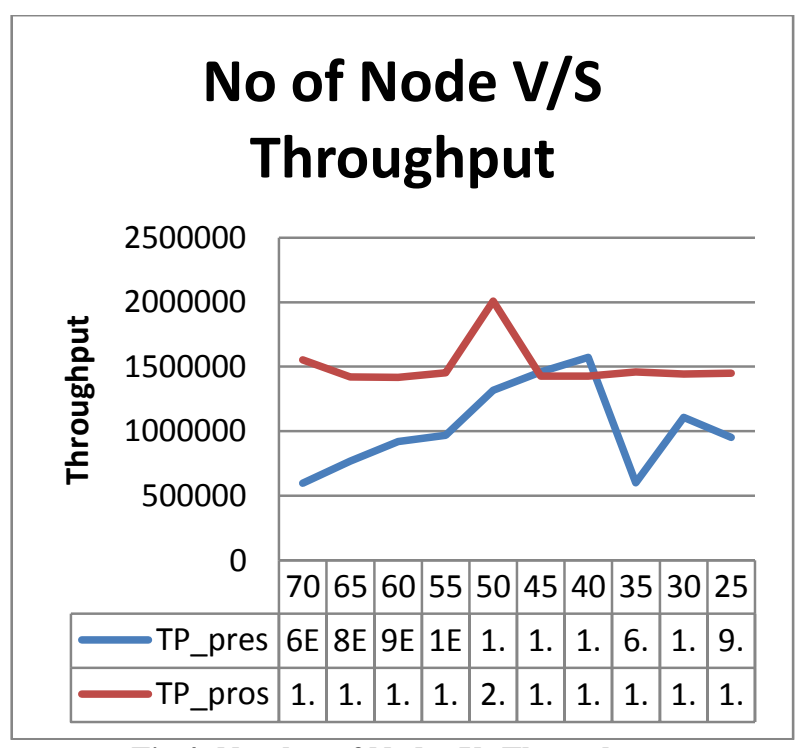

Fig 4: Number of Nodes Vs Throughput

Table 2: Throughput values with varying packet rate

\begin{tabular}{|c|c|c|}
\hline $\begin{array}{c}\text { Packet } \\
\text { rate }\end{array}$ & $\begin{array}{c}\text { ARS Throughput } \\
\text { in Bps }\end{array}$ & $\begin{array}{c}\text { ARS with fuzzy logic } \\
\text { Throughput in Bps }\end{array}$ \\
\hline 4 & $1.46 \mathrm{E}+06$ & $1.43 \mathrm{E}+06$ \\
\hline 8 & $1.48 \mathrm{E}+06$ & $1.54 \mathrm{E}+06$ \\
\hline
\end{tabular}




\begin{tabular}{|l|l|l|}
\hline 12 & $1.22 \mathrm{E}+06$ & $1.50 \mathrm{E}+06$ \\
\hline 16 & $1.19 \mathrm{E}+06$ & $1.51 \mathrm{E}+06$ \\
\hline 20 & $1.25 \mathrm{E}+06$ & $1.46 \mathrm{E}+06$ \\
\hline
\end{tabular}

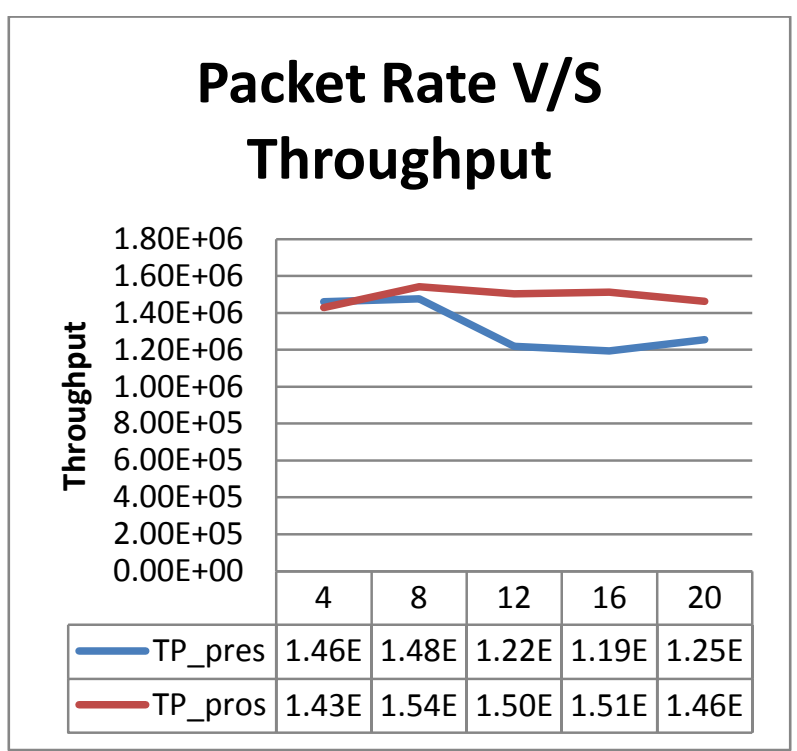

Fig 5: Packet Rate Vs Throughput

Table 3: Latency values with varying number of nodes

\begin{tabular}{|c|c|c|}
\hline Node & $\begin{array}{c}\text { ARS Latency in } \\
\text { sec }\end{array}$ & $\begin{array}{c}\text { ARS with fuzzy logic } \\
\text { Latency in sec }\end{array}$ \\
\hline 70 & 0.161703 & 0.0347751 \\
\hline 65 & 0.12561 & 0.037161 \\
\hline 60 & 0.0978817 & 0.0463789 \\
\hline 55 & 0.107934 & 0.0371178 \\
\hline 50 & 0.14444 & 0.0146746 \\
\hline 45 & $1.22 \mathrm{E}-01$ & 0.0260353 \\
\hline 40 & $4.78 \mathrm{E}-02$ & 0.0420598 \\
\hline 35 & $1.49 \mathrm{E}-01$ & 0.0399372 \\
\hline 30 & $1.28 \mathrm{E}-01$ & 0.0260025 \\
\hline 25 & $1.97 \mathrm{E}-01$ & 0.0395125 \\
\hline
\end{tabular}

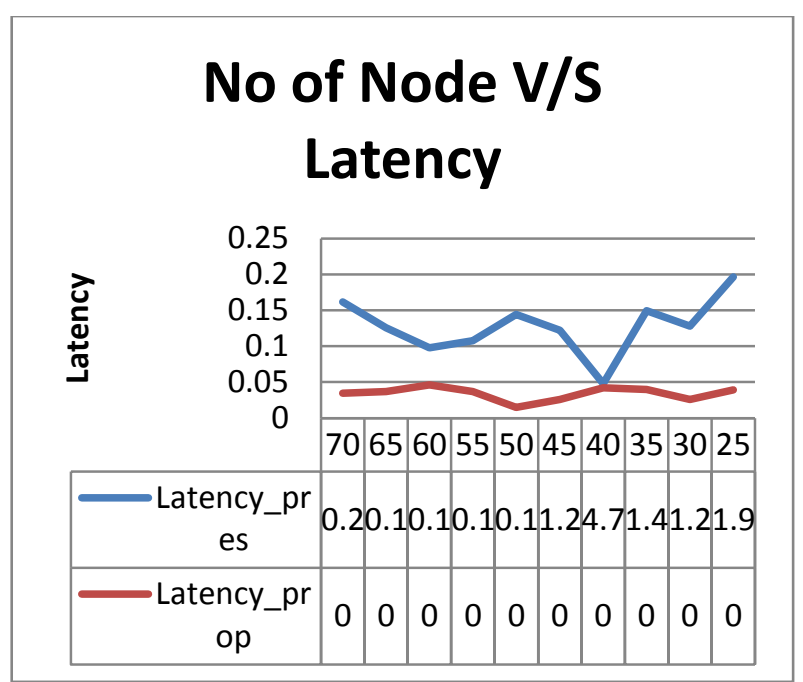

Fig 5: Number of Nodes Vs Latency

Table 4: Latency values with varying packet rate

\begin{tabular}{|c|c|c|}
\hline $\begin{array}{c}\text { Packet } \\
\text { rate }\end{array}$ & $\begin{array}{c}\text { ARS Latency in } \\
\text { sec }\end{array}$ & $\begin{array}{c}\text { ARS with Fuzzy Logic } \\
\text { Latency in sec }\end{array}$ \\
\hline 4 & $1.22 \mathrm{E}-01$ & $\mathbf{0 . 0 2 6 0 3 5 3}$ \\
\hline 8 & 0.0266494 & 0.0319115 \\
\hline 12 & 0.164407 & 0.0270232 \\
\hline 16 & 0.110854 & 0.0241179 \\
\hline 20 & 0.0293756 & 0.0293068 \\
\hline
\end{tabular}

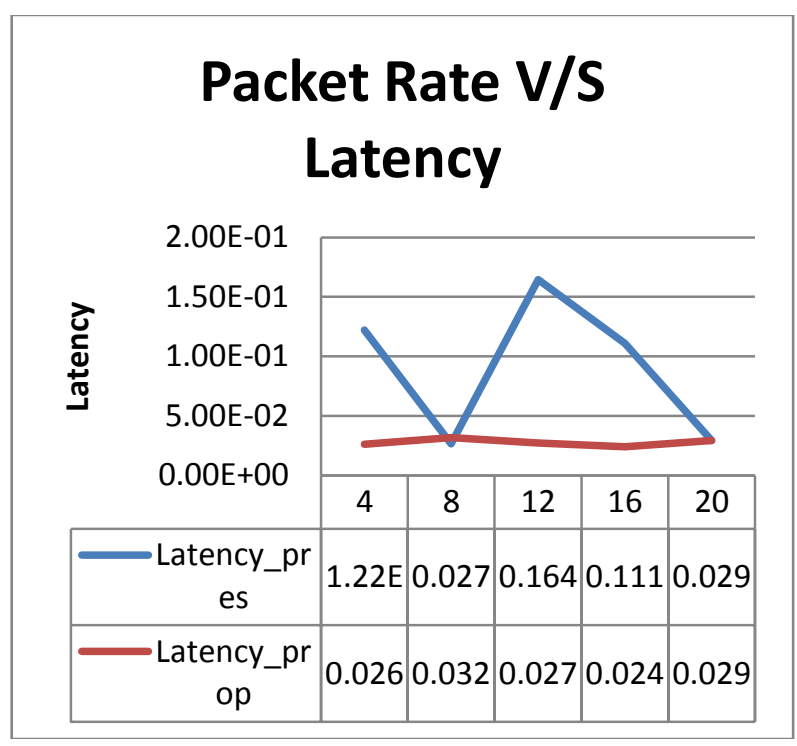

Fig 6: Packet Rate Vs Latency

\section{CONCLUSION}

Wireless mesh networks are generally used to provide seamless internet connection within low to medium topology changes. Generally such a network is conceived as more reliable due to fixed access points. However if the nodes are mobile, then their QoS and link quality of Access points keeps varying. Thus routing must be suitable enough to guarantee that there is no failure or minimum failure. In this system as cost based multipath routing is used, the QOS parameters lifetime, latency can be improved. We also offered automatic changing of paths, topology by handoff and by selecting 
alternative routes. This is called reconfiguration. Therefore QoS driven routing, automated reconfiguration and best path transmission ensures better network performance. By combining intelligent fuzzy based system we achieve better PDR in comparison to pure admission control system.

\section{FUTURE SCOPE}

Internet traffic is steadily growing due to increaing service demands and number of users. therefore power consumption might play a vital role for its futher growth. The system can be further improved by incorporating parameters like Energy of the nodes and link strength in the fuzzy decision system.

\section{REFERENCES}

[1] I. Akyildiz, X. Wang, and W. Wang, "Wireless mesh networks: A survey," Comput. Netw., vol. 47, no. 4, pp. 445-487, Mar. 2005.Ding, W. and Marchionini, G. 1997

[2] M. Alicherry, R. Bhatia, and L. Li, "Joint channel assignment and routing for throughput optimization in multi-radio wireless mesh networks," in Proc. ACM MobiCom, Cologne, Germany, Aug. 2005, pp. 58-72.

[3] yu-Han Kim and Kang G. Shin, IEEE/ACM TRANSACTIONS ON NETWORKING, "SelfReconfigurable Wireless Mesh Networks ”, APRIL 2011

[4] Mehajabeen Fatima, Roopam Gupta,R.Venkatesh , T.K. Bandhopadhyay " Route Discovery by Cross Layer Approach for MANET "International Journal of Computer Applications, January 2012.

[5] Len Malinin " On Application of Fuzzy Logic to Decisions Making in Solving Inventive Problems"“'International Journal of Computer and Information technology

[6] R. Draves, J. Padhye, and B. Zill, "Routing in multiradio, multi-hop wireless mesh networks," in Proc. ACM MobiCom 